\title{
Effects of metformin on clinical outcome in diabetic patients with advanced HCC receiving sorafenib
}

\author{
Andrea Casadei Gardini, Giorgia Marisi, Emanuela Scarpi, Mario Scartozzi, \\ Luca Faloppi, Nicola Silvestris, Gianluca Masi, Caterina Vivaldi, Oronzo \\ Brunetti, Stefano Tamberi, Francesco Giuseppe Foschi, Emiliano Tamburini, \\ Elena Tenti, Salvatore Ricca Rosellini, Paola Ulivi, Stefano Cascinu, Oriana \\ Nanni \& Giovanni Luca Frassineti
}

To cite this article: Andrea Casadei Gardini, Giorgia Marisi, Emanuela Scarpi, Mario Scartozzi, Luca Faloppi, Nicola Silvestris, Gianluca Masi, Caterina Vivaldi, Oronzo Brunetti, Stefano Tamberi, Francesco Giuseppe Foschi, Emiliano Tamburini, Elena Tenti, Salvatore Ricca Rosellini, Paola Ulivi, Stefano Cascinu, Oriana Nanni \& Giovanni Luca Frassineti (2015): Effects of metformin on clinical outcome in diabetic patients with advanced HCC receiving sorafenib, Expert Opinion on Pharmacotherapy, DOI: 10.1517/14656566.2015.1102887

To link to this article: http://dx.doi.org/10.1517/14656566.2015.1102887

Published online: 29 Oct 2015.

Submit your article to this journal

View related articles $\asymp$

View Crossmark data $\nearrow$ 


\section{EXPERT OPINION}

1. Introduction

2. Patients and methods

3. Results

4. Discussion

5. Conclusion

\section{Effects of metformin on clinical} outcome in diabetic patients with advanced HCC receiving sorafenib

\author{
Andrea Casadei Gardini ${ }^{\dagger}$, Giorgia Marisi, Emanuela Scarpi, Mario Scartozzi, \\ Luca Faloppi, Nicola Silvestris, Gianluca Masi, Caterina Vivaldi, \\ Oronzo Brunetti, Stefano Tamberi, Francesco Giuseppe Foschi, \\ Emiliano Tamburini, Elena Tenti, Salvatore Ricca Rosellini, Paola Ulivi, \\ Stefano Cascinu, Oriana Nanni \& Giovanni Luca Frassineti \\ ${ }^{\dagger}$ Department of Medical Oncology, Istituto Scientifico Romagnolo per lo Studio e Cura dei Tumori \\ (IRST) IRCCS, Meldola, Italy
}

Background and objective: Several studies have reported an association between type 2 diabetes mellitus and hepatocellular carcinoma (HCC). Data from several retrospective studies and meta-analyses have highlighted a reduction of about $50 \%$ in the risk of developing HCC in cirrhotic patients treated with metformin for diabetes. The aim of this study was to evaluate the different outcomes of patients who received or did not receive metformin during treatment with sorafenib.

Methods: We analyzed 93 patients consecutively treated with sorafenib. Forty-two (45.2\%) patients were diabetic, of whom 31 were on metformin. Progression-free survival (PFS) and overall survival (OS) were estimated with the Kaplan-Meier method and compared with the log-rank test.

Results: The concomitant use of sorafenib and metformin was associated with a median PFS of 2.6 months $(95 \% \mathrm{Cl} 1.9-3.3)$ compared to 5.0 months $(95 \% \mathrm{Cl} 2.5-8.2)$ for patients receiving sorafenib alone $(p=0.029)$. The median OS of patients treated with the combination was 10.4 months $(95 \%$ Cl 3.9-14.4) compared to 15.1 months (95\% Cl 11.7-17.8) for those who were not given metformin ( $p=0.014)$.

Conclusions: Our findings could be the result of increased tumor aggressiveness and resistance to sorafenib in metformin-treated patients.

Keywords: diabetes, hepatocellular carcinoma, insulin, metformin, sorafenib

Expert Opin. Pharmacother. [Early Online]

\section{Introduction}

Each year, >500,000 people worldwide are diagnosed with hepatocellular carcinoma (HCC), including about 20,000 new cases in the US.[1] It is the fifth most common cancer and the second most frequent cause of cancer-related death in men.[2] The incidence of HCC increases progressively with age in all populations, reaching its peak at 70 years.[3] Medical treatment remains one of the largest "black holes" in oncology as HCC is one of the most chemoresistant tumors, and no systemic drug was available for patients with advanced disease until 2007. Sorafenib, a multikinase inhibitor of the VEGFR, the platelet-derived growth factor receptor and Raf kinases, has been shown to increase median overall survival (OS); Llovet et al. reported a median OS of 7.9 months in a placebo group compared with 10.7 months in patients given sorafenib (hazard ratio $(\mathrm{HR})=0.69 ; 95 \%$ CI $0.55-0.87 ; \mathrm{p}=0.00058)$, representing a $31 \%$ decrease in the relative risk of death.[4] 
Metformin, a biguanide medication commonly used in patients with diabetes, is known to inhibit the mTOR pathway through AMP-activated protein kinase (AMPK) activation. It has also been shown to inhibit tumor growth in vitro and in vivo by inducing apoptosis in various malignancies including melanoma [5] and breast [6] and lung cancer.[7] The results from some retrospective studies suggest that metformin prevents the development of HCC in patients with diabetes [8,9] and in diabetic patients with chronic liver disease.[10] A large population-based study by Chen et al. [8] demonstrated a dose-dependent decrease in the risk of HCC among diabetic patients taking metformin, whereas a recent metaanalysis confirmed a decrease of $50 \%$ in the incidence of HCC among diabetics receiving the drug.[11] It has also been shown to have a potent tumor-suppressive effect in other malignancies through AMPK activation and subsequent inhibition. Conflicting data have emerged on the antiangiogenic action of metformin,[12] some studies indicating that it enhances angiogenesis,[13,14] upregulates VEGF and increases nitric oxide bioavailability in cardiovascular disease animal models, $[15,16]$ others suggesting that metformin downregulates several angiogenesis-related genes.[17]

The aim of our retrospective study was to evaluate the prognostic significance of metformin in patients with HCC treated with sorafenib.

\section{Patients and methods}

\subsection{Patient population}

This study was done using the medical records databases of our institute (IRST IRCCS), Università Politecnica delle Marche; Pisa University Hospital and the National Cancer Institute of Bari 'Giovanni Paolo II'. Ninety-three patients with HCC consecutively treated with sorafenib twice daily ( $2 \times 200 \mathrm{mg}$ tablets) between March 2008 and August 2014 were included in the study. Patients who had been taking insulin for at least 5 years at the time of the HCC diagnosis were considered "patients with diabetes treated with insulin," whereas those who had been on metformin at for at least 5 years when HCC was diagnosed were considered "patients with diabetes treated with metformin." None of the nondiabetic patients had ever received metformin. Patients were monitored closely for toxicity and dose reductions were made on the basis of type and severity of toxicity. Treatment with sorafenib was continued until disease progression, unacceptable toxicity or death occurred. Disease progression was assessed using Modified Response Evaluation Criteria in Solid Tumors criteria.

Data were entered into electronic data files by investigators from each participating center and were checked by the data management center (IRST) for missing information and internal consistency. The study protocol was reviewed and approved by local Ethics Committees. All patients provided written informed consent to take part in the study.

\subsection{Statistical analysis}

Descriptive data were reported as median with range for continuous variables, and absolute and relative frequencies for categorical variables. Progression-free survival (PFS) was calculated from the day of the start of treatment until the day of documented disease progression or last follow-up. OS was calculated from the day of the start of treatment until the day of death or last follow-up. Patients lost to follow-up were censored at the time of last contact.

PFS, OS and their 95\% CI were estimated using the Kaplan-Meier method and compared with the log-rank test. Estimated HRs and their 95\% CI were calculated from the Cox proportional hazard regression model. SAS statistical software version 9.3 (SAS Institute, Cary, NC, USA) was used for all statistical analyses and a p-value < 0.05 was considered statistically significant.

\section{Results}

Between March 2008 and August 2014, 93 patients diagnosed with HCC were consecutively treated with sorafenib. The case series comprised 74 men and 19 women with a median age of 71 years (range $28-87$ years). All patients had Child-Pugh A HCC. Eleven patients had Barcelona Clinic Liver Cancer Stage B (BCLC-B) HCC and 82 had BCLC-C HCC. Thirty-five $(32.55 \%)$ patients had previously undergone transarterial chemo embolization (TACE) (Table 1).

Table 1. Patient characteristics.

\begin{tabular}{lc}
\hline Characteristic & No. patients (\%) \\
\hline Median age, years (range) & $71(28-87)$ \\
Sex & \\
Male & $74(79,5)$ \\
Female & $19(20,5)$ \\
Etiology & \\
Viral & $67(72.0)$ \\
Alcoholic & $6(6.5)$ \\
NAFLD & $13(14.0)$ \\
Other & $7(7.5)$ \\
Diabetes & \\
No & $51(54.8)$ \\
Yes & $42(45.2)$ \\
Antidiabetic drug & $11(11.8)$ \\
Insulin & $31(33.3)$ \\
Metformin & $51(54.9)$ \\
None & $61(65.5)$ \\
ECOG & $27(29.6)$ \\
0 & $5(5.4)$ \\
1 &
\end{tabular}

NAFLD: non-alcoholic fatty liver disease, ECOG: Eastern Cooperative Oncology Group. 
The most common etiologies of liver disease were hepatitis C (34\%), alcoholic liver disease (29\%), fatty liver disease (15\%) and hepatitis B (9\%). Forty-two patients $(45.2 \%)$ were diabetic and $31(33.3 \%)$ were receiving metformin. The case series was homogeneous for age and sex but not for disease etiology. $25.4 \%$ of nondiabetic patients and $3.2 \%$ of diabetic patients were hepatitis B-positive (Table 2). The median PFS of all patients was 3.2 months (95\% CI: $2.5-5.0$ ) and median OS was 13.9 months (95\% CI: 10.4-15.8).

The concomitant use of sorafenib and metformin was associated with a median PFS of 2.6 months $(95 \% \mathrm{CI}$ : 1.9-3.3) compared with 5.0 months (95\% CI: 2.5-8.2) for patients not taking metformin $(p=0.029)$ (Table 3 and Figure 1A). Median OS of patients receiving the two drugs was 10.4 months (95\% CI: 3.9-14.4) compared with 15.1

Table 2. Homogeneity of cases analyzed.

\begin{tabular}{|c|c|c|c|c|}
\hline & \multirow{2}{*}{$\frac{\text { No. diabetes (51) }}{\text { No. (\%) }}$} & \multirow{2}{*}{$\begin{array}{l}\text { Diabetes + metformin (31) } \\
\text { No. (\%) }\end{array}$} & \multirow{2}{*}{$\frac{\text { Diabetes + insulin (11) }}{\text { No. (\%) }}$} & \multirow[t]{2}{*}{$\mathbf{p}$} \\
\hline & & & & \\
\hline \multicolumn{5}{|l|}{ Age, years } \\
\hline$\leq 40$ & $2(3)$ & $0(0)$ & $0(0)$ & \\
\hline$>40 \leq 60$ & $14(27.4)$ & $5(16.1)$ & $2(18.1)$ & \\
\hline$>60$ & $35(69.6)$ & $26(83.8)$ & $9(81.1)$ & 0.257 \\
\hline \multicolumn{5}{|l|}{ Sex } \\
\hline Male & $35(68.7)$ & $24(77.4)$ & $9(81.8)$ & \\
\hline Female & $16(31.3)$ & $7(22.6)$ & $2(18.2)$ & 0.237 \\
\hline \multicolumn{5}{|l|}{ Staging } \\
\hline BCLC B & $6(11.7)$ & $4(12.9)$ & $1(9)$ & 0.897 \\
\hline $\mathrm{BCLC} C$ & $45(88.3)$ & $27(87.1)$ & $10(91)$ & \\
\hline MELD score & & & & 0.954 \\
\hline$<10$ & $41(80.3)$ & $24(77.4)$ & $9(81.8)$ & \\
\hline$>10$ & $10(19.7)$ & $7(22.6)$ & $2(18.2)$ & \\
\hline$\alpha$-Fetoprotein & & & & 0.543 \\
\hline$<400$ & $40(78.4)$ & $22(70.9)$ & $8(72.7)$ & \\
\hline$>400$ & $11(21.6)$ & $9(29.1)$ & $3(27.3)$ & \\
\hline \multicolumn{5}{|l|}{ Etiology } \\
\hline Hepatitis C & $26(50.9)$ & $11(35.4)$ & $4(36.3)$ & \\
\hline Hepatitis B & $13(25.4)$ & $1(3.2)$ & $1(9)$ & \\
\hline Alcoholic liver disease & $5(9.8)$ & $7(22.5)$ & $2(18.1)$ & \\
\hline NAFLD & $3(5.8)$ & $10(32.2)$ & $2(18.1)$ & \\
\hline Other & $4(7.8)$ & $2(6.4)$ & $3(27.2)$ & 0.012 \\
\hline
\end{tabular}

BCLC: Barcelona Clinic Liver Cancer; NAFLD: non-alcoholic fatty liver disease; MELD: Model for End-Stage Liver Disease.

Table 3. Outcomes related to metformin and sorafenib.

\begin{tabular}{|c|c|c|c|c|c|c|c|}
\hline & \multirow[t]{2}{*}{ No. patients } & \multicolumn{3}{|c|}{ PFS } & \multicolumn{3}{|c|}{ os } \\
\hline & & No. events & $\begin{array}{l}\text { Median PFS } \\
(95 \% \mathrm{Cl})\end{array}$ & $\mathbf{p}$ & No. events & $\begin{array}{l}\text { Median OS } \\
(95 \% \mathrm{Cl})\end{array}$ & $\mathbf{p}$ \\
\hline \multicolumn{8}{|l|}{ Diabetes } \\
\hline No & 51 & 43 & $3.9(2.5-6.2)$ & & 25 & $14.9(11.7-17.8)$ & \\
\hline Yes & 42 & 37 & $2.6(2.0-4.6)$ & 0.226 & 27 & $11.2(5.8-15.8)$ & 0.198 \\
\hline \multicolumn{8}{|l|}{ Metformin } \\
\hline No & 62 & 52 & $5.0(2.5-8.2)$ & & 30 & $15.1(11.7-17.8)$ & \\
\hline Yes & 31 & 28 & $2.6(1.9-3.3)$ & 0.029 & 22 & $10.4(3.9-14.4)$ & 0.014 \\
\hline $\begin{array}{l}\text { Patients with diabetes treated with } \\
\text { insulin }\end{array}$ & 11 & 9 & $10.0(0.7-18.3)$ & & 5 & 17.6 (2.2-nr) & \\
\hline $\begin{array}{l}\text { Patients with diabetes treated with } \\
\text { metformin }\end{array}$ & 31 & 28 & $2.6(1.9-3.3)$ & 0.083 & 22 & $10.4(3.9-14.4)$ & 0.069 \\
\hline
\end{tabular}

OS: overall survival; PFS: progression-free survival. 
A

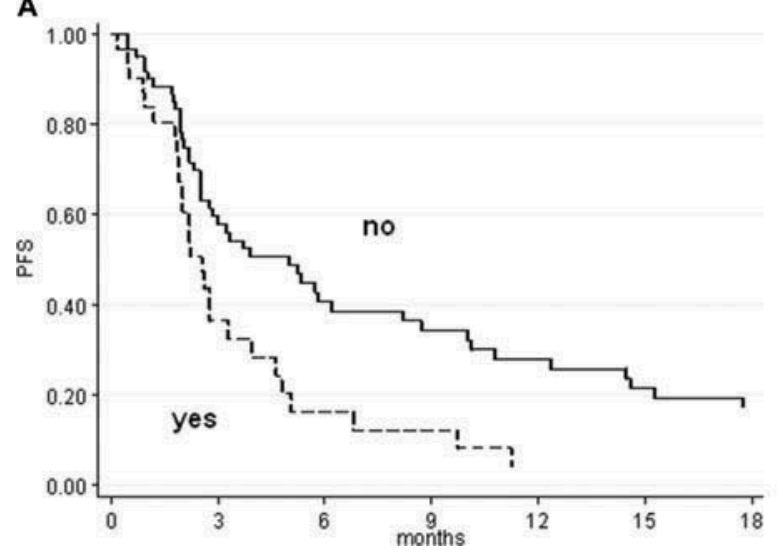

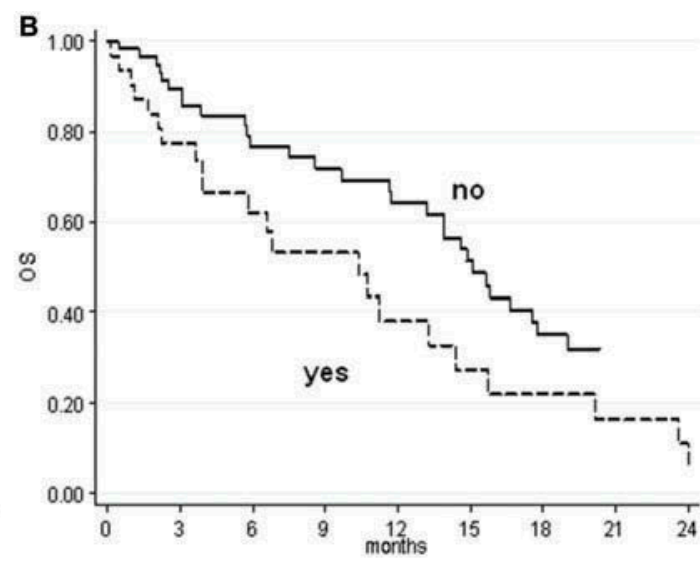

Figure 1. Kaplan-Meier curves of the overall population. (A) Progression-free survival according to metformin treatment. (B) Overall survival according to metformin treatment. The unbroken line shows patients who did not receive metformin, whereas the dashed line shows patients who were given metformin in combination with sorafenib. OS: overall survival; PFS: progression-free survival.

months (95\% CI: 11.7-17.8) for those taking sorafenib only $(\mathrm{p}=0.014)$ (Table 3 and Figure 1B).

In diabetic patients, the concomitant use of sorafenib and metformin was associated with a median PFS of 2.6 months (95\% CI: 1.9-3.3) compared with 10.0 months (95\% CI: 0.7-18.3) for those taking insulin ( $\mathrm{p}=0.083$ ) (Table 3 and Figure 2A). A median OS of 10.4 months (95\% CI: 3.914.4) was observed for patients taking sorafenib and metformin compared with 17.6 months (95\% CI: 2.2-nr) for those on insulin ( $\mathrm{p}=0.069)$ (Table 3 and Figure $2 \mathrm{~B})$.

The concomitant use of TACE and metformin was associated with a median PFS of 7.4 months (95\% CI: 4.3-15.7) compared with 11.2 (95\% CI: 3.4-15.7) for those not taking metformin $(\mathrm{p}=0.827)$. Median OS for these groups was

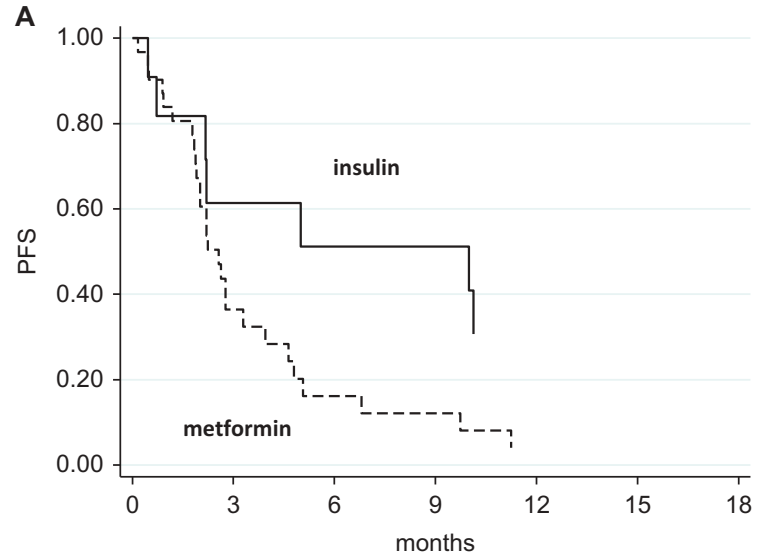

22.0 (95\% CI: 13.7-38.9) compared with 43.7 (95\% CI: $19.0-n r)$, respectively $(\mathrm{p}=0.05)$.

Considering the overall population, the risk of progression, adjusted by age, sex, smoking habits and etiology, was higher in diabetic patients taking metformin compared with those who were not diabetic (HR: 2.57; 95\% CI: 1.43-4.63; $\mathrm{p}=0.001)$. Patients with diabetes taking insulin had an adjusted risk of progression of 0.90 (95\% CI: 0.40-2.03; $\mathrm{p}=0.813)$ compared with non-diabetic cases. Similar results were observed with regard to survival (HR: 2.32; 95\% CI: 1.10-4.90; $\mathrm{p}=0.022$; and HR: 0.55; 95\% CI: 0.18-1.63; $\mathrm{p}=0.295$, respectively) (Table 4). Considering diabetic patients only, the risk of progression, adjusted by age, sex, smoking habits and etiology, was higher in patients taking

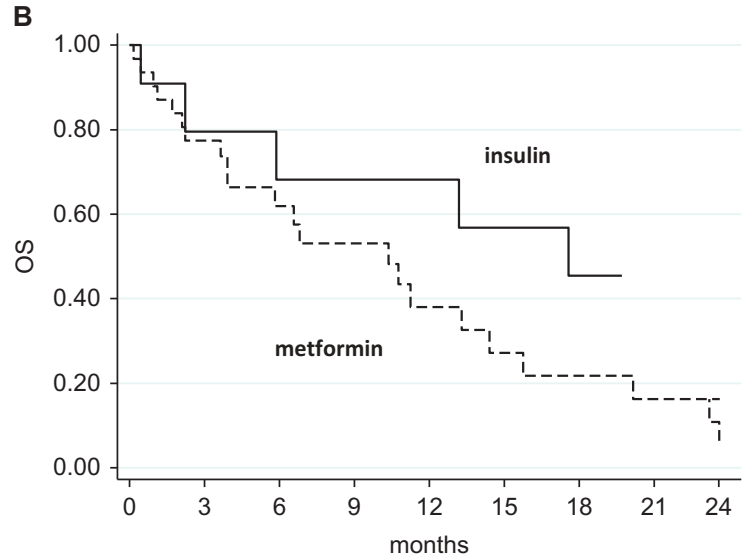

Figure 2. Kaplan-Meier curves of diabetic patients. (A) Progression-free survival. (B) Overall survival. The unbroken line shows diabetic patients who received insulin in combination with sorafenib, whereas the dashed line shows diabetic patients who underwent treatment with metformin and sorafenib. OS: overall survival; PFS: progression-free survival. 
Table 4. Hazard ratio of PFS and OS in diabetic patients receiving metformin or insulin.

\begin{tabular}{|c|c|c|c|c|}
\hline & \multicolumn{2}{|l|}{ PFS } & \multicolumn{2}{|l|}{ os } \\
\hline & HR $(95 \% \mathrm{Cl})$ & $\mathbf{p}$ & HR $(95 \% \mathrm{Cl})$ & $\mathbf{p}$ \\
\hline No diabetes & 1.00 & & 1.00 & \\
\hline Patients with diabetes treated with insulin & $0.90(0.40-2.03)$ & 0.813 & $0.55(0.18-1.63)$ & 0.295 \\
\hline Patients with diabetes treated with metformin & $2.57(1.43-4.63)$ & 0.001 & $2.32(1.10-4.90)$ & 0.022 \\
\hline
\end{tabular}

HR: hazard ratio; OS: overall survival; PFS: progression-free survival.

metformin than in those taking insulin (HR: 4.30; 95\% CI: $1.30-14.27 ; \mathrm{p}=0.017)$. Results were similar for survival (HR: 5.16; 95\% CI: 1.53-17.63; $\mathrm{p}=0.008$ ).

\section{Discussion}

Type 2 diabetes is a significant risk factor for the development of malignancies, including HCC.[10,11] $\mathrm{Li}$ et al. recently described a significant linear trend in HCC incidence with increasing glycated hemoglobin levels,[18] but other authors have reported conflicting data on this.[19-24] Metformin has garnered considerable interest as a chemopreventive and chemotherapeutic agent given the increased risk of HCC among patients with insulin resistance.

As far as we know, this is the first work to evaluate the association between metformin and sorafenib in patients with HCC. Several studies have shown that metformin decreases the risk of HCC in patients with cirrhosis. [8,9] We considered patients who developed HCC during therapy with metformin and who later received first-line treatment with sorafenib. From a clinical point of view, metformin did not enhance the activity of sorafenib. Patients receiving the twodrug combination showed poorer PFS and OS than those given sorafenib alone. The overall difference of 2.4 months in median PFS and 4.7 months in median OS between patients taking metformin and those not taking metformin was significant. Our results also highlighted a distinct, albeit not significant, advantage in terms of response, PFS and OS in diabetic patients taking insulin. Larger case series are needed to confirm these data. The patients analyzed were homogeneous for age, sex, stage, score Model for End-Stage Liver Disease score and basal levels of $\alpha$-fetoprotein and lactate dehydrogenase, but not for etiology. Previous studies have shown a lower activity of sorafenib in $\mathrm{HBsAg}$-positive patients.[25] Had our case series been homogeneous, the difference would probably have been greater. We also noted that response to TACE was not affected by metformin administration, which confirms that sorafenib-resistant cancer clones are generated during the development of HCC in patients taking metformin.

We do not have information on baseline glucose or HbA1C levels and this could be a limitation to the study, but we think that having of well-controlled or not well-controlled diabetes before sorafenib treatment would not have made any difference to our results.

Molecular alterations in protein kinases involved in the molecular action of metformin may lead to a different response to the sorafenib-metformin combination. The molecular action of metformin is mainly associated with the inhibition of the mTOR pathway, which plays an important role in metabolism, growth and proliferation of cancer cells. [26] mTOR inhibition may be exerted by metformin through AMPK activation, which is pivotal in the energy homeostasis process.[27,28] AMPK is activated through AMP binding or through phosphorylation by molecular components such as the liver kinase B1 (LKB1), the ataxiatelangiectasia mutated (ATM) protein kinase or the calcium activated kinase (CAMKK2).[29] Although ATM, LKB1 and AMPK are not the direct targets of metformin, the drug specifically inhibits mitochondrial respiratory chain complex I activities,[30] inducing an increase in the cellular AMP: ATP ratio, which activates AMPK.[30] There is evidence, however, that metformin also inhibits the mTOR pathway in an AMPK-independent manner.[6,31] Both AMPK-dependent and -independent pathways are thought to mediate the anticancer effects of metformin treatment. Moreover, the antiangiogenic effect of metformin have been attributed to both AMPK-dependent and -independent mechanisms.[17]

Similar mechanisms of action have been described for sorafenib. In particular, Groenendijk et al. demonstrated that sorafenib, such as metformin, acts as an activator of AMPK through LKB1 or CAMKK2. The authors reported that patients with $K R A S$-mutated advanced NSCLC receiving metformin during sorafenib treatment showed better disease control than those taking sorafenib alone. They also observed a synergistic growth inhibition of NSCLC cells in vitro and in vivo with the sorafenib-metformin combination, as well as synergistic AMPK activation and downstream mTOR pathway inhibition. [32] Sorafenib would thus seem to use the same activation pathway as metformin, that is, AMPK.

Conversely, we observed that diabetic patients already taking metformin before the start of treatment with sorafenib were more resistant to the multikinase inhibitor than those with insulin-treated diabetes. A possible explanation for these contradictory results is that tumors developing during chronic treatment with metformin are more likely to have 
intrinsic mechanisms of resistance to metformin, which may also lead to resistance to sorafenib.

\section{Conclusion}

In conclusion, although somewhat limited by its retrospective nature, our study was based on consecutively selected patients, thus reducing the risk of bias. Findings may be attributable to increased tumor aggressiveness and resistance to sorafenib in metformin-treated patients. Molecular characterization of patients will enable us to identify a subset of HCC patients who are more resistant to sorafenib. We also plan to validate these data in a prospective study including non-diabetic patients.

\section{Acknowledgement}

The authors thank Ursula Elbling for editing the manuscript.

\section{Declaration of interest}

The author has no relevant affiliations or financial involvement with any organization or entity with a financial interest in or financial conflict with the subject matter or materials discussed in the manuscript. This includes employment, consultancies, honoraria, stock ownership or options, expert testimony, grants or patents received or pending, or royalties.

\section{Bibliography}

Papers of special note have been highlighted as either if interest $(\bullet)$ or of considerable interest $(\bullet \bullet)$ to readers.

1. Seeff LB, Hoofnagle JH. Epidemiology of hepatocellular carcinoma in areas of low hepatitis B and hepatitis C endemicity. Oncogene. 2006;25:3771-3777.

2. El-Serag HB, Mason AC, Key C. Trends in survival of patients with hepatocellular carcinoma between 1977 and 1996 in the United States. Hepatology. 2001;33:62-65.

3. El-Serag HB. Hepatocellular carcinoma: Recent trends in the United States. Gastroenterology. 2004;127(5 Suppl 1): S27-34.

4. Llovet JM, Ricci S, Mazzaferro V, et al. Sorafenib in advanced hepatocellular carcinoma. N Engl J Med. 2008;359:378-390.

5. Tomic $\mathrm{T}$, Botton $\mathrm{T}$, Cerezo $\mathrm{M}$, et al. Metformin inhibits melanoma development through autophagy and apoptosis mechanisms. Cell Death Dis. 2011;2: e199.

6. Zakikhani M, Dowling R, Fantus IG, et al. Metformin is an AMP kinasedependent growth inhibitor for breast cancer cells. Cancer Res. 2006;66:10269-10273.

7. Memmott RM, Mercado JR, Maier CR, et al. Metformin prevents tobacco carcinogeninduced lung tumorigenesis. Cancer Prev Res (Phila). 2010;3:1066-1076.

8. Chen HP, Shieh JJ, Chang CC, et al. Metformin decreases hepatocellular carcinoma risk in a dose-dependent manner: population-based and in vitro studies. Gut. 2013;62:606-615.
-• This interesting clinical study showed that metformin is active in hepatocellular carcinoma (HCC), reducing the risk of this tumor in diabetic patients.

9. Chen TM, Lin CC, Huang PT, et al. Metformin associated with lower mortality in diabetic patients with early stage hepatocellular carcinoma after radiofrequency ablation. J Gastroenterol Hepatol. 2011;26:858-865.

10. Donadon V, Balbi M, Mas MD, et al. Metformin and reduced risk of hepatocellular carcinoma in diabetic patients with chronic liver disease. Liver Int. 2010;30:750-758.

11. Singh S, Singh PP, Singh AG, et al. Antidiabetic medications and the risk of hepatocellular cancer: A systematic review and meta-analysis. Am J Gastroenterol. 2013;108:881-891. quiz 892.

-• This is a meta-analysis showed that patients taking metformin have a lower risk of HCC.

12. Tan BK, Adya R, Chen J, et al. Metformin decreases angiogenesis via NF-kappaB and Erk1/2/Erk5 pathways by increasing the antiangiogenic thrombospondin-1. Cardiovasc Res. 2009;83:566-574.

13. Ziche M, Morbidelli L. Molecular regulation of tumor angiogenesis by nitric oxide. Eur Cytokine Netw. 2009;20:164-170.

14. Martin MJ, Hayward R, Viros A, et al. Metformin accelerates the growth of BRAF V600E-driven melanoma by upregulating VEGF-A. Cancer Discov. 2012;2:344-355.

15. Cittadini A, Napoli R, Monti MG, et al. Metformin prevents the development of chronic heart failure in the SHHF rat model. Diabetes. 2012;61:944-953.

16. Sena CM, Matafome P, Louro $\mathrm{T}$, et al. Metformin restores endothelial function in aorta of diabetic rats. Br J Pharmacol. 2011;163:424-437.

17. Dallaglio K, Bruno A, Cantelmo AR, et al. Paradoxic effects of metformin on endothelial cells and angiogenesis. Carcinogenesis. 2014;35:1055-1066.

-. Key paper that assesses the effects of metformin on endothelial cells and describes an antiangiogenic activity of metformin in vitro and in vivo.

18. Li CI, Chen HJ, Lai HC, et al. Hyperglycemia and chronic liver diseases on risk of hepatocellular carcinoma in Chinese patients with type 2 diabetes-National cohort of Taiwan Diabetes Study. Int J Cancer. 2015;136:2668-2679.

19. Sluik D, Boeing H, Montonen J, et al. $\mathrm{HbAlc}$ measured in stored erythrocytes is positively linearly associated with mortality in individuals with diabetes mellitus. Plos One. 2012;7:e38877.

20. Dabrowski M. Glycated hemoglobin, diabetes treatment and cancer risk in type 2 diabetes. A case-control study. Ann Agric Environ Med. 2013;20:116-121.

21. Yang $\mathrm{X}$, Wang $\mathrm{Y}$, Luk $\mathrm{AO}$, et al. Enhancers and attenuators of risk associations of chronic hepatitis B virus infection with hepatocellular carcinoma in type 2 diabetes. Endocr Relat Cancer. 2013;20:161-171.

22. Donadon V, Balbi M, Valent F, et al. Glycated hemoglobin and antidiabetic strategies as risk factors for hepatocellular 
carcinoma. World J Gastroenterol. 2010;16:3025-3032.

23. de Beer JC, Liebenberg L. Does cancer risk increase with $\mathrm{HbA1c}$, independent of diabetes? BJC. 2014;110:2361-2368.

24. Yang X, Ko GT, So WY, et al. Associations of hyperglycemia and insulin usage with the risk of cancer in type 2 diabetes: the Hong Kong diabetes registry. Diabetes. 2010;59:1254-1260.

25. Bruix J, Raoul JL, Sherman M, et al. Efficacy and safety of sorafenib in patients with advanced hepatocellular carcinoma: subanalyses of a phase III trial. J Hepatol. 2012;57:821-829.

26. Chiang GG, Abraham RT. Targeting the mTOR signaling network in cancer. Trends Mol Med. 2007;13:433-442.

27. Gong L, Goswami S, Giacomini KM, et al. Metformin pathways: pharmacokinetics and pharmacodynamics. Pharmacogenet Genomics. 2012;22:820-827.

- Interesting study that describes in a detailed manner a metformin pathway.

28. Hardie DG. The LKB1-AMPK pathwayfriend or foe in cancer? Cancer Cell. 2013;23:131-132.
29. Foretz M, Viollet B. Regulation of hepatic metabolism by AMPK. J Hepatol. 2011;54:827-829.

30. Hardie DG. Neither LKB1 nor AMPK are the direct targets of metformin. Gastroenterology. 2006;131:973. author reply 974-975.

31. Kalender A, Selvaraj A, Kim SY, et al. Metformin, independent of AMPK, inhibits mTORC1 in a rag GTPasedependent manner. Cell Metab. 2010;11:390-401.

32. Groenendijk FH, Mellema WW, van der Burg E, et al. Sorafenib synergizes with metformin in NSCLC through AMPK pathway activation. Int $\mathrm{J}$ Cancer. 2015;136:1434-1444.

-. This is one of the first reports showing the effect of multikinase inhibitor sorafenib in NSCLC patients receiving metformin and identified a synergistic effect of both drugs.

Affiliation

Andrea Casadei Gardini ${ }^{\dagger 1}$, Giorgia Marisi ${ }^{2}$, Emanuela Scarpi ${ }^{3}$, Mario Scartozzi ${ }^{4}$, Luca Faloppi $^{5}$, Nicola Silvestris ${ }^{6}$, Gianluca Masi ${ }^{7}$, Caterina Vivaldi ${ }^{7}$, Oronzo Brunetti ${ }^{6}$, Stefano Tamberi ${ }^{8}$, Francesco Giuseppe Foschi ${ }^{9}$, Emiliano Tamburini ${ }^{10}$, Elena Tenti ${ }^{11}$, Salvatore Ricca
Rosellini $^{12}$, Paola Ulivi ${ }^{2}$, Stefano Cascinu ${ }^{5}$, Oriana Nanni ${ }^{3} \&$ Giovanni Luca Frassineti ${ }^{1}$

${ }^{\dagger}$ Authors for correspondence

${ }^{1}$ Department of Medical Oncology, Istituto Scientifico Romagnolo per lo Studio e Cura dei Tumori (IRST) IRCCS, Meldola, Italy

${ }^{2}$ Biosciences Laboratory, IRST IRCCS, Meldola, Italy

${ }^{3}$ Unit of Biostatistics and Clinical Trials, IRST IRCCS, Meldola, Italy

${ }^{4}$ Department of Medical Oncology, University of Cagliari, Cagliari, Italy

${ }^{5}$ Department of Clinical Medical Oncology, AO

Ospedali Riuniti, Università Politecnica delle

Marche, Ancona, Italy

${ }^{6}$ Medical Oncology Unit, National Cancer

Institute 'Giovanni Paolo II', Bari, Italy

${ }^{7}$ Department of Oncology, Pisa University

Hospital, Pisa, Italy

${ }^{8}$ Department of Oncology, Degli Infermi

Hospital, Faenza, Italy

${ }^{9}$ Department of Internal Medicine, Degli Infermi

Hospital, Faenza, Italy

${ }^{10}$ Department of Oncology, Infermi Hospital,

Rimini, Italy

${ }^{11}$ Oncology Pharmacy Laboratory, IRST IRCCS, Meldola, Italy

${ }^{12}$ Unit of Gastroenterology, Morgagni-Pierantoni Hospital, Forli, Italy

Tel: +39-0543-739970;

Fax: +39-0543-739249;

E-mail: andrea.casadei@irst.emr.it 